\title{
RESCALED INTERACTING DIFFUSIONS CONVERGE TO SUPER BROWNIAN MOTION
}

\author{
By J. TheOdore COX AND ACHIM KLENKE ${ }^{1}$ \\ Syracuse University and Universität zu Köln
}

Super Brownian motion is known to occur as the limit of properly rescaled interacting particle systems such as branching random walk, the contact process and the voter model.

In this paper we show that certain linearly interacting diffusions converge to super Brownian motion if suitably rescaled in time and space. The results comprise nearest neighbor interaction as well as long range interaction.

\section{Introduction.}

1.1. Motivation. Super Brownian motion was first derived as the high-density, short-lifetime diffusion limit of branching Brownian motions. See [5] for a survey. More recently it has also been found that particle systems where particles have less independence than in branching processes, namely the contact process and the voter model, have super Brownian motion as diffusion limit. This is particularly interesting because the local activity in these processes is heavily dependent on the (local) density of particles. For dimension $d=1$ Mueller and Tribe [8] show that the contact process can be rescaled to a super Brownian motion with a drift that depends on the local intensity of particles. On the other hand the limit of the onedimensional voter model is a super Brownian motion where the local branching rate is a decreasing function $\gamma(\theta)=1-\theta$ of the local intensity $\theta \in[0,1]$ of particles.

In higher dimensions the dependence on the local density of particles gets washed out and in the limit the actual intensity of particles has to be replaced by its expected value. See [6] and [2] for the results on the contact process and the voter model, respectively.

1.2. Our model. The model that we study in this paper is that of linearly coupled diffusions indexed by $\mathbb{Z}^{d}, d \geq 3$. More precisely, we consider the process $\left(X_{t}\right)_{t \geq 0}$ that takes values in a suitable subspace $\mathfrak{X}$ of $[0, \infty)^{\mathbb{Z}^{d}}$ and that is the unique strong solution of the stochastic differential equation

$$
d X_{t}=\mathcal{A} X_{t} d t+\sqrt{g\left(X_{t}\right)} d B_{t},
$$

Received January 2002; revised April 2002.

${ }^{1}$ Supported in part by the DFG.

AMS 2000 subject classifications. Primary 60K35, 60G57; secondary 60F05, 60J80, 60H10.

Key words and phrases. Martingale problem, spatially rescaled particle systems, diffusion limit, long range limit. 
where $\mathcal{A}$ is the $q$-matrix of a random walk on $\mathbb{Z}^{d}$, and

$$
\begin{array}{ll}
g:[0, \infty) \rightarrow[0, \infty) & \text { is locally Lipschitz continuous, } \\
g^{-1}((0, \infty))=(0, b) & \text { for some } b \in(0, \infty], \\
g(z) \leq C\left(1+z^{2}\right) & \text { for some } C<\infty
\end{array}
$$

Finally, $\left\{\left(B_{t}(i)\right)_{t \geq 0}, i \in \mathbb{Z}^{d}\right\}$ is an independent family of standard Brownian motions. If $b<\infty$, then $\mathfrak{X}=[0, b]^{\mathbb{Z}^{d}}$ is the natural choice. If $b=\infty$, we have to be a bit more careful. In this case we let $\mathfrak{X}$ be a Liggett-Spitzer space with respect to $\mathcal{A}$. (Essentially this is a subset of $[0, \infty) \mathbb{Z}^{d}$ with a polynomial growth condition. As we do not deal with this case here, we do not describe the LiggettSpitzer space in detail but only refer to [7].) It is well known (see [10]) that, under these conditions, for $X_{0} \in \mathfrak{X}$ there exists a unique strong solution of (1.1) that assumes values in $\mathfrak{X}$.

Since we want to rescale this process to super Brownian motion we have to assume that $\mathcal{A}$ has finite variance. For simplicity of notation we will assume that the random walk generated by $\mathcal{A}$ is driftless and the coordinates are uncorrelated with the same variance:

$$
\sum_{i \in \mathbb{Z}^{d}} \mathcal{A}(0, i) i_{\alpha} i_{\beta}=\sigma^{2} \mathbb{1}_{\alpha=\beta}, \quad \alpha, \beta \in\{1, \ldots, d\} .
$$

We now rescale the space and the diffusion speed as well as the mass of the particles. To this end define for $N \in \mathbb{N}$ and $t \geq 0$ the random measure

$$
X_{t}^{N}=\frac{1}{N} \sum_{i \in \mathbb{Z}^{d}} X_{t N}(i) \delta_{i / \sqrt{N}},
$$

where $\delta$ denotes the Dirac measure. Further let $\mathcal{M}_{f}\left(\mathbb{R}^{d}\right)$ denote the space of finite Borel measures on $\mathbb{R}^{d}$ equipped with the vague topology. The idea is that if the initial state converges to a finite measure

$$
X_{0}^{N} \rightarrow \mu \in \mathcal{M}_{f}\left(\mathbb{R}^{d}\right) \quad \text { as } N \rightarrow \infty
$$

then $X^{N}$ also converges as $N \rightarrow \infty$ to super Brownian motion with some branching rate $\gamma$. The next step is to define this branching rate $\gamma$ in terms of the ingredients for $X$. To this end we have to make the additional assumption that $b<\infty$ and that $g$ is of the Wright-Fisher form

$$
g(x)=\kappa x\left(1-\frac{x}{b}\right)^{+}, \quad x \geq 0,
$$

for some $\kappa>0$.

Note that the assumptions we have made imply that for every $\theta \geq 0$ there exists a unique stationary ergodic invariant measure $v_{\theta}^{b, \kappa}$ for $X$ with intensity $\theta$, that is,

$$
\int v_{\theta}^{b, \kappa}(d x) x(i)=\theta, \quad i \in \mathbb{Z}^{d} .
$$


See [4]. Denote by $q$ the probability that a random walk with symmetrized $q$-matrix

$$
\hat{\mathcal{A}}:=\frac{1}{2}\left(\mathcal{A}+\mathcal{A}^{T}\right)
$$

does not return to the origin after first leaving it. This probability is positive since $\mathcal{A}$ is transient, and it can be expressed as $q=(-\hat{G}(0,0) \hat{\mathcal{A}}(0,0))^{-1}$, where $(\exp (t \hat{\mathcal{A}}))_{t \geq 0}$ is the semigroup of an $\hat{\mathcal{A}}$-random walk and $\hat{G}$ is its Green function

$$
\hat{G}(i, j)=\int_{0}^{\infty} \exp (t \hat{\mathcal{A}})(i, j) d t, \quad i, j \in \mathbb{Z}^{d} .
$$

This can easily be seen using the following argument: Let $Z$ be random walk with $q$-matrix $\hat{\mathcal{A}}$ starting in $Z_{0}=0$ and define $\tau_{0}:=\inf \left\{t>0: Z_{t} \neq 0\right\}, \tau_{1}:=\inf \{t>$ $\left.\tau_{0}: Z_{t}=0\right\}$. Hence a simple renewal argument shows

$$
\begin{aligned}
\hat{G}(0,0)=\mathbf{E}\left[\int_{0}^{\infty} \mathbb{1}_{Z_{t}=0} d t\right] & =\mathbf{E}\left[\tau_{0}\right]+\mathbf{P}\left[\tau_{1}<\infty\right] \mathbf{E}\left[\int_{0}^{\infty} \mathbb{1}_{Z_{t}=0} d t\right] \\
& =(-\hat{\mathcal{A}}(0,0))^{-1}+(1-q) \hat{G}(0,0) .
\end{aligned}
$$

LEMMA 1.1. The limit

$$
\gamma:=\gamma^{b, \kappa}:=\lim _{\theta \downarrow 0} \theta^{-1} \int v_{\theta}(d x) g(x(0))
$$

exists and is equal to

$$
\gamma^{b, \kappa}=\frac{2 b q \kappa}{2 b q+\kappa} .
$$

We prove this lemma in Section 2 by a straightforward computation using the duality of interacting Wright-Fisher diffusions to coalescing random walks. The main point about the assumption that $g$ is of the Wright-Fisher type is that we could not establish (1.9) by other means [though for $g(x)=\gamma x$ it is trivial]. In other cases, as for example $g(x)=x^{2}\left((b-x)^{+}\right)^{2}$, one can show that $\gamma=0$.

To formulate our first theorem let $Y^{\gamma, \sigma^{2}}$ denote super Brownian motion in $\mathbb{R}^{d}$ with branching rate $\gamma$ and (spatial) diffusion constant $\sigma^{2}>0$. That is, for $Y_{0}^{\gamma, \sigma^{2}}=\mu \in \mathcal{M}_{f}\left(\mathbb{R}^{d}\right), Y^{\gamma, \sigma^{2}}$ is the unique solution of the martingale problem: for $\varphi \in C_{0}^{2}\left(\mathbb{R}^{d}\right)$,

$$
M_{t}^{\varphi}:=Y_{t}^{\gamma, \sigma^{2}}(\varphi)-\int_{0}^{t} Y_{s}^{\gamma, \sigma^{2}}\left(\frac{\sigma^{2}}{2} \Delta \varphi\right) d s
$$

is a continuous square-integrable martingale with square variation process

$$
\left\langle M^{\varphi}\right\rangle_{t}=\int_{0}^{t} Y_{s}^{\gamma, \sigma^{2}}\left(\gamma \varphi^{2}\right) d s
$$

(see [5]). We suspect that the statement of the following theorem is true for all $g$ fulfilling (1.2). However, we could show it only for $g$ of the Wright-Fisher form. 
THEOREM 1. Under the assumptions (1.3), (1.5) and (1.6), and with $\gamma$ from (1.10), the rescaled process $X^{N}$ converges as $N \rightarrow \infty$ in distribution on the Skorohod space $D\left([0, \infty): \mathcal{M}_{f}\left(\mathbb{R}^{d}\right)\right)$ to $Y^{\gamma, \sigma^{2}}$.

Note that the voter model is the limit of interacting Wright-Fisher diffusions with $b=1$ and $\kappa \rightarrow \infty$. Letting $\kappa \rightarrow \infty$ in (1.10) leads in fact to the same branching rate for the limiting super Brownian motion as established for the voter model in [2]. On the other hand, letting $b \rightarrow \infty$ with fixed $\kappa$ one might expect to end up with the same branching rate that one gets from rescaling interacting Feller's branching diffusions $(b=\infty, g(x)=\kappa x)$, namely with $\kappa$. This is in fact true since $\gamma^{b, \kappa} \rightarrow \kappa$ as $b \rightarrow \infty$.

REMARK 1.2. Without the spatial rescaling one might wonder whether there is convergence to super-random walk $\bar{Y}^{\gamma}$ with some branching rate $\gamma$ and jump matrix $\mathcal{A}^{T}$. This is in fact true if one defines $\bar{X}_{t}^{N}=\sum_{i \in \mathbb{Z}^{d}} N X_{t}(i) \delta_{i}$. If $g$ fulfills (1.2) and in addition is differentiable in 0 with $g^{\prime}(0)=\gamma$, then $\bar{X}^{N} \rightarrow \bar{Y}^{\gamma}$ as $N \rightarrow \infty$. This statement can easily be shown using the comparison technique of [3] and a truncation argument. In fact, using the fact that $\sup _{z>0} \frac{g(z)}{z}<\infty$ we can bound the variance of the total mass $\left\|X_{T}^{N}\right\|$ for every fixed $T$. Using Doob's inequality we get that for every $\varepsilon>0$ there is a $K<\infty$ such that

$$
\mathbf{P}\left[\sup _{N \geq 1} \sup _{t \in[0, T]}\left\|X_{t}^{N}\right\|>K\right]<\varepsilon .
$$

Hence we can change $g$ to

$$
\tilde{g}(z):= \begin{cases}g(z), & z \leq \frac{K}{N}, \\ \frac{N}{K} g\left(\frac{K}{N}\right) z, & z \geq \frac{K}{N},\end{cases}
$$

and with high probability $\bar{X}^{N}$ and the corresponding $\tilde{X}^{N}$ coincide up to time $T$. Now define $\gamma^{N,+}=\sup _{z \in(0, K / N)} \frac{g(y)}{y}, \gamma^{N,-}=\inf _{z \in(0, K / N)} \frac{g(y)}{y}$ and $g^{N, \pm}(z)=$ $\gamma^{N, \pm} z$. Hence $g^{N,-} \leq \tilde{g}^{N} \leq g^{N,+}$. The comparison scheme of [3] now yields that, for a certain distribution determining class of continuous functionals $F$ of $\left(X_{t}\right)_{t \in[0, T]}$,

$$
\mathbf{E}\left[F\left(\bar{Y} \gamma^{N,-}\right)\right]=\mathbf{E}\left[F\left(\tilde{X}^{N,-}\right)\right] \leq \mathbf{E}\left[F\left(\tilde{X}^{N}\right)\right] \leq \mathbf{E}\left[F\left(\tilde{X}^{N,+}\right)\right]=\mathbf{E}\left[F\left(\bar{Y}^{\gamma^{N,+}}\right)\right]
$$

and $\tilde{\gamma} \mapsto \mathbf{E}[F(\bar{Y} \tilde{\gamma})]$ is monotone. As $\gamma^{N,+} \downarrow \gamma$ and $\gamma^{N,-} \uparrow \gamma$ one gets

$$
\lim _{N \rightarrow \infty} \mathbf{E}\left[F\left(\tilde{X}^{N}\right)\right]=\mathbf{E}\left[F\left(\bar{Y}^{\gamma}\right)\right] .
$$

Thus $\tilde{X}^{N} \rightarrow \bar{Y}^{\gamma}$ as $N \rightarrow \infty$, and hence also $\bar{X}^{N} \rightarrow \bar{Y}^{\gamma}$ as $N \rightarrow \infty$. 
1.3. The long range model. We would have liked to formulate our Theorem 1 for more general functions $g$; however, we could not establish Lemma 1.1 for the general case. One way to overcome this problem is to change the scaling of the model such that the range of interaction gets larger and larger and the limit of the equilibria $v_{\theta}^{N}$ can be described via the mean field equation.

That is, in the limit $N \rightarrow \infty$ under $v_{\theta}^{N}(d x)$ the coordinates of $x$ are independent and are distributed according to the unique invariant measure $\bar{v}_{\theta}^{c, g}$ of the onedimensional diffusion

$$
d Z_{t}=c\left(\theta-Z_{t}\right) d t+\sqrt{g\left(Z_{t}\right)} d B_{t} .
$$

Here $c>0$ is a constant that reflects the strength of the interaction. Note that $Z_{t}$ solves the integral equation

$$
Z_{t}=\theta+\int_{0}^{t} e^{c(s-t)} \sqrt{g\left(Z_{s}\right)} d B_{s}+e^{-c t}\left(Z_{0}-\theta\right)
$$

The idea is that any given coordinate interacts with so many other coordinates that a law of large numbers applies. Note that $\bar{v}_{\theta}^{c, g}$ has a density that can be computed explicitly,

$$
\frac{\bar{v}_{\theta}^{c, g}(d z)}{d z}=C_{\theta}^{c, g} \frac{1}{g(z)} \exp \left(-2 c \int_{\theta}^{z} \frac{r-\theta}{g(r)} d r\right) .
$$

Here $C_{\theta}^{c, g}$ is a normalizing constant. To see that this is the equilibrium density, note that $Z$ has generator $g f(z)=c(\theta-z) f^{\prime}(z)+\frac{1}{2} g(z) f^{\prime \prime}(z)$ with adjoint $g^{*} f(z)=c \frac{d}{d z}((\theta-z) f(z))+\frac{d^{2}}{d z^{2}}(g(z) f(z))$ and compute

$$
g^{*} \frac{d \bar{v}_{\theta}^{c, g}(d z)}{d z}=0
$$

Using (1.14) we see that $\mathbf{E}^{\theta}\left[Z_{t}\right]=\theta$ for all $t \geq 0$ and thus $\mathbf{E}^{\bar{v}_{\theta}^{c, g}}[Z]=\theta$. Using (1.14) again we get

$$
\operatorname{Var}^{\bar{v}_{\theta}^{c, g}}\left[Z_{0}\right]=\operatorname{Var}^{\bar{v}_{\theta}^{c, g}}\left[Z_{t}\right]=\int_{0}^{t} e^{2 c(t-s)} \mathbf{E}^{\bar{v}_{\theta}^{c, g}}\left[g\left(Z_{s}\right)\right] d s+e^{-2 c t} \operatorname{Var}^{\bar{v}_{\theta}^{c, g}}\left[Z_{0}\right] .
$$

Using stationarity we can let $t \rightarrow \infty$ and get

$$
\operatorname{Var}^{\bar{v}_{\theta}^{c, g}}\left[Z_{0}\right]=\int_{0}^{\infty} e^{-2 c s} d s \mathbf{E}^{\bar{v}_{\theta}^{c, g}}\left[g\left(Z_{0}\right)\right]
$$

Thus

$$
\int \bar{v}_{\theta}^{c, g}(d z)(z-\theta)^{2}=\frac{1}{2 c} \int \bar{v}_{\theta}^{c, g}(d z) g(z) .
$$

The explicit form of $\bar{v}_{\theta}^{c, g}$ allows us to show that $\gamma$ can be defined as in Lemma 1.1. We quote the following lemma from Baillon et al. ([1], Proposition 5, 
the uniformity that we state here is not in [1] but follows very easily from their argument).

LEMMA 1.3. The limit

$$
\gamma^{c, g}:=\lim _{\theta \downarrow 0} \theta^{-1} \int \bar{v}_{\theta}^{c, g}(d z) g(z)
$$

exists and $\gamma^{c, g}>0$ if and only if

$$
\int_{0}^{b / 2} \frac{z}{g(z)} d z<\infty
$$

In this case the limit in (1.17) is uniform in c on compact subsets of $(0, \infty)$ and

$$
\gamma^{c, g}=2 c \int_{0}^{b} \exp \left(-2 c \int_{0}^{y} \frac{z}{g(z)} d z\right) d y .
$$

In the case where $g$ is of the Wright-Fisher type it is simple to compute $\gamma^{c, g}$ explicitly.

COROLlary 1.4. If $g(x)=\kappa x(1-x / b)^{+}$, then $\gamma^{c, g}=2 c b \kappa /(\kappa+2 c b)$.

We will henceforth assume that that (1.18) holds. Note that this is the case, for example, if $g$ has a positive derivative at 0 or if $g(x) \sim x^{1+\beta}$ as $x \rightarrow 0$ for some $\beta \in[0,1)$. On the other hand, for $g(x)=x^{2}\left((b-x)^{+}\right)^{2}$ the condition is violated.

Let us now define the long range models $\bar{X}^{N}$. Let $\left(M_{N}\right)_{N \in \mathbb{N}}$ be a sequence in $\mathbb{N}$ that increases to $\infty$. This sequence is arbitrary but will be kept fixed. Let $\sigma^{2}>0$ and define

$$
\mathcal{A}^{N}(i, j)=3 \sigma^{2}\left[\left(2 M_{N}+1\right)^{-d} \mathbb{1}_{\left\{-M_{N}, \ldots, M_{N}\right\}^{d}}(j-i)-\mathbb{1}_{\{0\}}(j-i)\right] .
$$

That is, $\mathcal{A}^{N}$ is the $q$-matrix of a rate $3 \sigma^{2}$ [more precisely, $3 \sigma^{2}\left(1-\left(2 M_{N}+1\right)^{-d}\right)$ ] random walk that jumps to each point in distance at most $M_{N}$ with equal probability. For $\varphi: \mathbb{R}^{d} \rightarrow \mathbb{R}$ define

$$
\tilde{\mathcal{A}}^{N} \varphi(x)=N \sum_{j \in \mathbb{Z}^{d}} \mathcal{A}^{N}(0, j) \varphi\left(x+N^{-1 / 2} M_{N}^{-1} j\right) .
$$

LEMMA 1.5. For $\varphi \in C_{0}^{2}\left(\mathbb{R}^{d}\right)$ and $x \in \mathbb{R}^{d}$,

$$
\lim _{N \rightarrow \infty}\left\|\tilde{\mathcal{A}}^{N} \varphi-\frac{\sigma^{2}}{2} \Delta \varphi\right\|_{\infty}=0 .
$$

Now define $\bar{X}^{N}$ as the solution of (1.1) but with $\mathcal{A}$ replaced by $\mathcal{A}^{N}$ and let

$$
X_{t}^{N}=\frac{1}{N} \sum_{i \in \mathbb{Z}^{d}} \bar{X}_{t N}^{N}(i) \delta_{i /\left(M_{N} \sqrt{N}\right)}
$$


Again we could establish the following theorem only when $g$ is of the WrightFisher form where we can exploit the well-known duality of interacting WrightFisher diffusions to coalescing random walks (see [9], Lemma 2.3). We give more details in the next section. However, here also we suspect that the statement is true for $g$ fulfilling only (1.2).

THEOREM 2. Under conditions (1.5) and (1.6) with $\gamma=\gamma^{3 \sigma^{2}, g}$,

$$
\mathcal{L}\left[X^{N}\right] \Rightarrow \mathcal{L}^{\mu}\left[Y^{\gamma, \sigma^{2}}\right] \quad \text { as } N \rightarrow \infty .
$$

1.4. Outline. In the next two sections we give the proofs of Theorems 1 and 2, respectively. The proofs rely on the duality of $X$ to coalescing random walks and make use of the ideas and statements developed in [2].

2. Proof of Theorem 1. Before we come to the proof of the theorem we prove the lemma that precedes it.

ProOf OF LEMMA 1.1. If we include for the moment the dependence of $X$ on the parameters $b$ and $\kappa$ in the notation we can write the following scaling property:

$$
\mathcal{L}^{x}\left[X^{b, \kappa}\right]=\mathcal{L}^{x / b}\left[b X^{1, \kappa / b}\right] .
$$

The verification is elementary and is omitted here. Note that we can conclude from (2.1) that $\gamma^{b, \kappa}=b \gamma^{1, \kappa / b}$. Hence it suffices to show the lemma for $b=1$.

Let us recall that the $n$th moments of interacting Wright-Fisher diffusions can be computed via a duality relation with a system of $n$ coalescing random walks. See [9], Lemma 2.3, for a full account of this. We need here only the first and second moments. Let $Z^{1}$ and $Z^{2}$ be random walks with $q$-matrix $\mathcal{A}^{T}$ that coalesce at rate $\kappa$ when they occupy the same site. Then the duality yields

$$
\begin{aligned}
\mathbf{E}\left[X_{t}\left(z^{1}\right)\right] & =\mathbf{E}^{z^{1}}\left[X_{0}\left(Z_{t}^{1}\right)\right]=\mathbf{E}\left[e^{t \mathcal{A}^{T}} X_{0}\left(z^{1}\right)\right], \\
\mathbf{E}\left[X_{t}\left(z^{1}\right) X_{t}\left(z^{2}\right)\right] & =\mathbf{E}^{\left(z^{1}, z^{2}\right)}\left[X_{0}\left(Z_{t}^{1}\right) X_{0}\left(Z_{t}^{2}\right)\right] .
\end{aligned}
$$

In particular, for $X_{0} \equiv \theta$

$$
\operatorname{Var}^{\theta}\left[X_{t}(0)\right]=\theta(1-\theta) \mathbf{P}^{(0,0)}\left[Z_{t}^{1} \neq Z_{t}^{2}\right]
$$

Letting $t \rightarrow \infty$ we get

$$
\operatorname{Var}^{v_{\theta}}\left[X_{0}(0)\right]=\theta(1-\theta) \mathbf{P}\left[Z^{1} \text { and } Z^{2} \text { do not coalesce }\right] \text {. }
$$


This latter probability can be computed in terms of the escape probability of $q$ of the difference walk (which has $q$-matrix $2 \hat{\mathcal{A}}$ ) as $\frac{2 q}{2 q+\kappa}$. Hence we have

$$
\begin{aligned}
\theta^{-1} \int v_{\theta}(d x) g\left(x_{0}\right) & =\frac{\kappa}{\theta} \int v_{\theta}(d x) x(0)(1-x(0)) \\
& =\kappa\left(1-\frac{1}{\theta} \int v_{\theta}(d x) x(0)^{2}\right) \\
& =\kappa\left(1-\theta-\frac{1}{\theta} \operatorname{Var}^{v_{\theta}}\left[X_{0}(0)\right]\right) \\
& =\frac{2 q \kappa}{2 q+\kappa}(1-\theta) .
\end{aligned}
$$

This clearly implies the assertion of the lemma.

PROOF OF THEOREM 1. The strategy of the proof is to describe the process $X^{N}$ via a martingale problem and to show that the quadratic variation process converges to that of super Brownian motion. Here we make use of the duality of interacting Wright-Fisher diffusions to coalescing random walks. In fact, the proof is quite similar to the one given in [2] for the voter model and we carry out in detail only the part that differs.

For ease of notation write

$$
\mathcal{A}^{N} \varphi(x)=N \sum_{i \in \mathbb{Z}^{d}} \varphi(x+i / \sqrt{N}) \mathcal{A}(0, i)
$$

and note that, for $\varphi \in C_{0}^{2}\left(\mathbb{R}^{d}\right)$,

$$
\mathcal{A}^{N} \varphi(x) \rightarrow \frac{\sigma^{2}}{2} \Delta \varphi(x) \quad \text { as } N \rightarrow \infty .
$$

To meet the technical requirements of [2] we will assume that $\varphi \in C_{0}^{3}\left(\mathbb{R}^{d}\right)$, in which case the convergence in (2.5) is uniform if the second and third derivatives of $\varphi$ are bounded (see [2], Lemma 2.6): if we let

$$
\begin{aligned}
\|\varphi\|_{2,3}:= & \sup \left\{\frac{d^{2}}{d x_{i} d x_{j}} \varphi(x), i, j=1, \ldots, d, x \in \mathbb{R}^{d}\right\} \\
& +\sup \left\{\frac{d^{3}}{d x_{i} d x_{j} d x_{k}} \varphi(x), i, j, k=1, \ldots, d, x \in \mathbb{R}^{d}\right\},
\end{aligned}
$$

then

$$
\lim _{N \rightarrow \infty} \sup _{\|\varphi\|_{2,3}<K}\left\|\mathcal{A}^{N} \varphi-\frac{\sigma^{2}}{2} \Delta \varphi\right\|_{\infty}=0, \quad K<\infty .
$$


We abbreviate

$$
\begin{aligned}
\Gamma_{s}^{N} & :=\sum_{x \in \mathbb{Z}^{d} / \sqrt{N}} N^{-1} g\left(N X_{s}^{N}(\{x\})\right) \delta_{x} \\
& =\sum_{i \in \mathbb{Z}^{d}} N^{-1} g\left(N X_{s N}(i)\right) \delta_{i / \sqrt{N}}
\end{aligned}
$$

It is an exercise in stochastic calculus to check that

$$
M_{t}^{\varphi, N}:=X_{t}^{N}(\varphi)-X_{0}^{N}(\varphi)-\int_{0}^{t} X_{s}^{N}\left(\mathcal{A}^{N} \varphi\right) d s
$$

is a continuous square-integrable martingale with quadratic variation process

$$
\left\langle M^{\varphi, N}\right\rangle_{t}=\int_{0}^{t} \Gamma_{s}^{N}\left(\varphi^{2}\right) d s .
$$

With a view to $(1.11),(1.12)$ and (2.4) it is clear that the main point is to show that

$$
\left\langle M^{\varphi, N}\right\rangle_{t}-\int_{0}^{t} \gamma X_{s}^{N}\left(\varphi^{2}\right) d s \rightarrow 0 \quad \text { as } N \rightarrow \infty .
$$

More precisely, the convergence has to be shown to take place in $L^{2}$.

In fact, the martingale problem has exactly the form of Theorem 2.1 of [2] with their error term $\varepsilon_{s}^{N}$ being zero and with their $V_{N, s}^{\prime}(x)$ replaced by $\frac{1}{2} N^{-1} g\left(N X_{s}^{N}(\{x\})\right)$. We proceed as in Section 4 of [2] and define, for $K>0$,

$$
\varepsilon_{K, \varphi}^{N, \gamma}(t):=\sup \left\{\left|\mathbf{E}\left[\left(\Gamma_{t}^{N}-\gamma X_{t}^{N}\right)\left(\varphi^{2}\right)\right]\right|: X_{0}^{N}(1) \leq K\right\} .
$$

We have to show the following lemma.

LEMMA 2.1 (Convergence of the mean).

$$
\lim _{N \rightarrow \infty} \varepsilon_{K, \varphi}^{N, \gamma}(t)=0
$$

For the proof of our Theorem 1, convergence of the means of the quadratic variation process is not enough but $L^{2}$-convergence is needed. Together with the following moment bounds Theorem 4.1 of [2] improves (2.10) to $L^{2}$-convergence and in fact yields the conclusion of the proof of Theorem 1.

LEMmA 2.2 (Moment bounds). Fix $T>0$. There exists a constant $C_{T}<\infty$ such that, for all $s \in[0, T]$,

$$
\begin{aligned}
\mathbf{E}\left[\Gamma_{s}^{N}(1)\right] & \leq C_{T} X_{0}^{N}(1), \\
\mathbf{E}\left[X_{s}^{N}(1)^{3}\right] & \leq C_{T}\left(X_{0}^{N}(1)^{3}+1\right), \\
\mathbf{E}\left[X_{s}^{N}(1)^{2} \Gamma_{s}^{N}(1)\right] & \leq C_{T}\left(X_{0}^{N}(1)^{3}+1\right) .
\end{aligned}
$$


PROOF. Let $L$ denote the Lipschitz constant of $g$. Then

$$
\Gamma_{s}^{N}(1) \leq L X_{s}^{N}(1) \text {. }
$$

Hence (2.11) holds with $C_{T}=L$. Also (2.13) holds with $C_{T}$ replaced by $L C_{T}$. Now note that Itô's formula yields

$$
\frac{d}{d s} \mathbf{E}\left[X_{s}^{N}(1)^{3}\right] \leq 3 L \mathbf{E}\left[X_{s}^{N}(1)^{2}\right]
$$

and

$$
\frac{d}{d s} \mathbf{E}\left[X_{s}^{N}(1)^{2}\right] \leq L \mathbf{E}\left[X_{s}^{N}(1)\right]=L X_{0}^{N}(1)
$$

Thus

$$
\mathbf{E}\left[X_{s}^{N}(1)^{3}\right] \leq X_{0}^{N}(1)^{3}+3 L T X_{0}^{N}(1)^{2}+\frac{3}{2} L^{2} T^{2} X_{0}^{N}(1) .
$$

Of course, $C_{T}$ can be chosen such that (2.12) holds.

PROOF OF LEMMA 2.1. Note that so far we could adopt the arguments of [2] without using the fact that $g$ is of the Wright-Fisher form. We will need it only here (and in Lemma 1.1) to exploit the duality to coalescing random walks in order to show (2.10).

Recall that

$$
g(x)=\kappa x(1-x / b)^{+} \quad \text { and } \quad \gamma=\frac{2 b q \kappa}{2 b q+\kappa},
$$

where $q$ is the escape probability of an $\hat{\mathcal{A}}$-random walk [recall (1.8)]. Recall also from the proof of Lemma 1.1 that we can use a scaling argument so that without loss of generality we may assume $b=1$. Hence we have

$$
\varepsilon_{K, \varphi}^{N, \gamma}(t)=\kappa \sup \left\{\mid \mathbf{E}\left[\sum _ { x \in \mathbb { Z } ^ { d } / \sqrt { N } } \left(\left(1-\frac{\gamma}{\kappa}\right) X_{t}^{N}(\{x\})\right.\right.\right.
$$

$$
\left.\left.\left.-N X_{t}^{N}(\{x\})^{2}\right) \varphi^{2}(x)\right] \mid: X_{0}^{N}(1) \leq K\right\} .
$$

Now let $Z^{1}$ and $Z^{2}$ be random walks with $q$-matrix $\mathcal{A}^{T}$ that coalesce at rate $\kappa$ when they are at the same site. Denote by $p_{t}=e^{t \mathcal{A}^{T}}$ the transition probability of $Z^{1}$. Let $A_{t}$ be the event that $Z^{1}$ and $Z^{2}$ have coalesced by time $t$ and $A:=\bigcup_{t \geq 0} A_{t}$. The duality yields [see (2.3) (with $Z^{1}=Z^{2}=i$ )]

$$
\begin{aligned}
N \mathbf{E}\left[X_{t}^{N}(\{i / \sqrt{N}\})^{2}\right]= & N^{-1} \mathbf{E}\left[X_{t N}(i)^{2}\right] \\
= & N^{-1} \mathbf{E}^{i}\left[X_{0}\left(Z_{t N}^{1}\right) ; A_{t N}\right] \\
& +N^{-1} \mathbf{E}^{i}\left[X_{0}\left(Z_{t N}^{1}\right) X_{0}\left(Z_{t N}^{2}\right) ; A_{t N}^{c}\right] .
\end{aligned}
$$


Using the central limit theorem, there exists a constant $C<\infty$ such that

$$
p_{t}(0, j) \leq\left(t^{-d / 2} \wedge 1\right) \cdot C, \quad j \in \mathbb{Z}^{d}, t>0 .
$$

Thus, since $X_{0}^{N}(1) \leq K$, the second term on the right-hand side is smaller than

$$
N^{-1} \mathbf{E}^{i}\left[X_{0}\left(Z_{t N}^{1}\right)\right]^{2} \leq N K^{2} \sup _{j \in \mathbb{Z}^{d}} p_{t N}(0, j)^{2} \leq C t^{-d} K^{2} \cdot N^{1-d} .
$$

Hence we have

$$
\sum_{i \in \mathbb{Z}^{d}} N^{-1} \mathbf{E}^{i}\left[X_{0}\left(Z_{t N}^{1}\right)\right]^{2} \varphi(i / \sqrt{N})^{2} \leq C C_{\varphi} K^{2} t^{-d} N^{1-d / 2},
$$

where the constant $C_{\varphi}$ depends on $\varphi$ only. Hence by dominated convergence it suffices to show that, for all $i \in \mathbb{Z}^{d}$,

$$
\begin{aligned}
\widetilde{\varepsilon}_{K}^{N, \gamma, i}(t):=N^{d / 2-1} \sup \{\mid & \mathbf{E}^{i}\left[X_{0}\left(Z_{t N}^{1}\right) ; A_{t N}^{c}\right] \\
\left.-\frac{\gamma}{\kappa} \mathbf{E}^{i}\left[X_{0}\left(Z_{t N}^{1}\right)\right] \mid: X_{0}(1) \leq K N\right\} & \rightarrow 0 \\
& \text { as } N \rightarrow \infty .
\end{aligned}
$$

However, this is true since (see the proof of Lemma 1.1)

$$
\lim _{T \rightarrow \infty} \mathbf{P}\left[A_{T}^{c}\right]=1-\mathbf{P}[A]=\frac{\gamma}{\kappa}
$$

and the distribution of $Z_{t N}^{1}$ and the conditional distribution of $Z_{t N}^{1}$ given $A_{t N}^{c}$ are close. To make this precise, let $\delta>0$ be arbitrary. Fix $T_{0}>0$ such that [with $C$ as in (2.16)]

$$
\left|\mathbf{P}\left[A_{T}^{c}\right]-\frac{\gamma}{\kappa}\right| \leq \frac{(t / 2)^{d / 2}}{C} \delta \quad \text { for all } T \geq T_{0} .
$$

Estimating the total amount of time two independent random walks spend together gives a bound on the probability that $Z^{1}$ and $Z^{2}$ coalesce between times $T$ and $t N$ and end in a particular point $j$,

$$
\begin{aligned}
\mathbf{P}^{i}\left[A_{t N}\right. & \left.\cap A_{T}^{c} \cap\left\{Z_{t N}^{1}=j\right\}\right] \\
& \leq \kappa \int_{T}^{t N} d r \sum_{k \in \mathbb{Z}^{d}} p_{r}(i, k) p_{r}(i, k) p_{t N-r}(k, j) \\
& \leq \kappa p_{t N}(i, j) \int_{T}^{t N} d r \sup _{k} p_{r}(0, k) \\
& \leq \kappa C^{2} t^{-d / 2} N^{-d / 2} \int_{T}^{t N} r^{-d / 2} d r \\
& \leq \frac{2 \kappa C^{2} t^{-d / 2}}{d-2} T^{1-d / 2} N^{-d / 2}
\end{aligned}
$$


Hence, by choosing $T_{0}$ large enough, in addition to (2.21) we may assume

$$
\sup _{j \in \mathbb{Z}^{d}} \mathbf{P}^{i}\left[A_{t N} \cap A_{T}^{c} \cap\left\{Z_{t N}^{1}=j\right\}\right] \leq \delta N^{-d / 2}, \quad T \geq T_{0} .
$$

Let $R>0$ be such that

$$
\mathbf{P}^{i}\left[\left|Z_{T_{0}}^{1}\right|>R\right]<\frac{\delta}{(1+(\gamma / \kappa))\left(1+(2 / t)^{d / 2} C\right)} .
$$

Using (2.16) and the Markov property at time $T_{0}$ we get, for $N \geq 2 T_{0} / t$,

$$
\left(1+\frac{\gamma}{\kappa}\right) \mathbf{P}^{i}\left[\left|Z_{T_{0}}^{1}\right|>R ; Z_{t N}^{1}=j\right] \leq \delta N^{-d / 2} .
$$

Using the central limit theorem again we get that there exists an $N_{0} \geq 2 T_{0} / t$ such that, for all $N \geq N_{0}$ and $|k|<R$,

$$
\left|p_{t N-T_{0}}(k, j)-p_{t N-T_{0}}(0, j)\right|<\frac{\delta}{1+(\gamma / \kappa)} N^{-d / 2} .
$$

Combining (2.23), (2.25), (2.26), (2.16), (2.24) and (2.21) and using the Markov property we get, for $N \geq N_{0}$,

$$
\begin{aligned}
&\left|\mathbf{P}^{i}\left[Z_{t N}^{1}=j ; A_{t N}^{c}\right]-\frac{\gamma}{\kappa} \mathbf{P}^{i}\left[Z_{t N}^{1}=j\right]\right| \\
& \leq\left|\mathbf{P}^{i}\left[Z_{t N}^{1}=j ; A_{T_{0}}^{c}\right]-\frac{\gamma}{\kappa} \mathbf{P}^{i}\left[Z_{t N}^{1}=j\right]\right|+\delta N^{-d / 2} \\
& \leq\left|\sum_{|k|<R} \mathbf{P}^{i}\left[Z_{t N}^{1}=j ; Z_{T_{0}}^{1}=k ; A_{T_{0}}^{c}\right]-\frac{\gamma}{\kappa} \mathbf{P}^{i}\left[Z_{t N}^{1}=j ; Z_{T_{0}}^{1}=k\right]\right| \\
&+2 \delta N^{-d / 2} \\
&=\left|\sum_{|k|<R} p_{t N-T_{0}}(k, j)\left(\mathbf{P}^{i}\left[Z_{T_{0}}^{1}=k ; A_{T_{0}}^{c}\right]-\frac{\gamma}{\kappa} \mathbf{P}^{i}\left[Z_{T_{0}}^{1}=k\right]\right)\right| \\
&+2 \delta N^{-d / 2} \\
& \leq\left|\mathbf{P}^{i}\left[\left|Z_{T_{0}}^{1}\right|<R ; A_{T_{0}}^{c}\right]-\frac{\gamma}{\kappa} \mathbf{P}^{i}\left[\left|Z_{T_{0}}^{1}\right|<R\right]\right| \cdot p_{t N-T_{0}}(0, j) \\
&+3 \delta N^{-d / 2} \\
& \leq\left|\mathbf{P}^{i}\left[A_{T_{0}}^{c}\right]-\frac{\gamma}{\kappa}\right| \cdot C\left(\frac{2}{t}\right)^{d / 2} N^{-d / 2}+4 \delta N^{-d / 2} \\
& \leq 5 \delta N^{-d / 2} .
\end{aligned}
$$

Since the estimate holds for all $j$, we get by Hölder's inequality

$$
\limsup _{N \rightarrow \infty} \widetilde{\varepsilon}_{K}^{N, \gamma, i}(t) \leq 5 K \delta .
$$


Since $\delta>0$ was arbitrary, (2.19) follows and the proof of Lemma 2.1 is complete.

3. Proof of Theorem 2. As in the proof of Theorem 1 we may assume without loss of generality that $b=1$. The proof here is analogous to the proof of Theorem 1. First we formulate the martingale problem. For $\varphi \in C_{0}^{2}\left(\mathbb{R}^{d}\right)$,

$$
M_{t}^{\varphi, N}:=X_{t}^{N}(\varphi)-X_{0}^{N}(\varphi)-\int_{0}^{t} X_{s}^{N}\left(\mathcal{A}^{N} \varphi\right)
$$

is a continuous square-integrable martingale with quadratic variation process

$$
\left\langle M^{\varphi, N}\right\rangle_{t}=\int_{0}^{t} \Gamma_{s}^{N}\left(\varphi^{2}\right) d s
$$

where

$$
\Gamma_{s}^{N}:=\sum_{x \in \mathbb{Z}^{d} / \sqrt{N} M_{N}} N^{-1} g\left(N X_{s}^{N}(\{x\})\right) \delta_{x} .
$$

By Lemma 1.5, $\tilde{\mathcal{A}}^{N} \varphi \rightarrow \frac{\sigma^{2}}{2} \Delta \varphi$ and hence again it is enough to show that, in $L^{2}$,

$$
\left\langle M^{\varphi, N}\right\rangle_{t}-\int_{0}^{t} \gamma X_{s}^{N}\left(\varphi^{2}\right) d s \rightarrow 0 \quad \text { as } N \rightarrow \infty .
$$

Using Theorem 4.1 of [2] it is enough to establish convergence of the means instead or, more precisely, Lemmas 2.1 and 2.2 in this setting. The proof of Lemma 2.1 works here without changes. In the proof of Lemma 2.2 we only need the central limit theorem (which is in force here, too) and the fact that the probability that two random walks do not coalesce is $\frac{\gamma}{\kappa}$. Here we work with two random walks $Z^{N, 1}$ and $Z^{N, 2}$ that run independently according to the $q$-matrix $\mathcal{A}^{N}$ and coalesce at rate $\kappa$ when they are at the same site. By $p_{t}^{N}=e^{t \mathcal{A}^{N}}$ we denote the transition probability of any of these random walks. Hence if $Z_{0}^{N, 1}=i, Z_{0}^{N, 2}=j$ and $i \neq j$, then [recall that the walks have rate $3 \sigma^{2}(1-$ $\left.\left.\left(2 M_{N}+1\right)^{-d}\right)\right]$

$$
\begin{aligned}
\mathbf{P}^{i, j}\left[Z^{N, 1} \text { and } Z^{N, 2} \text { never coalesce }\right] & \geq \mathbf{P}^{i, j}\left[Z_{t}^{N, 1} \neq Z_{t}^{N, 2} \text { for all } t>0\right] \\
& =1-\mathbf{P}^{0}\left[Z_{t}^{N, 1}=j-i \text { for some } t>0\right] \\
& \geq 1-3 \sigma^{2} \int_{0}^{\infty} p_{t}^{N}(0, j-i) d t .
\end{aligned}
$$

By the central limit theorem there exists a constant $C$ such that

$$
p_{t}^{N}(0, j-i) \leq C M_{N}^{-d}\left(t^{-d / 2} \wedge 1\right), \quad t>0, N \in \mathbb{N} .
$$

Thus, for $i \neq j$,

$$
\lim _{N \rightarrow \infty} \inf _{i \neq j} \mathbf{P}^{i, j}\left[Z^{N, 1} \text { and } Z^{N, 2} \text { never coalesce }\right]=1 .
$$


On the other hand, for $i=j$, the probability of coalescence before either $Z^{N, 1}$ or $Z^{N, 2}$ makes a first jump is $\frac{\kappa}{6 \sigma^{2}+\kappa}$. After the first jump, the probability of coalescence is negligible by (3.4). Thus, for $t>0$,

(3.5) $\lim _{N \rightarrow \infty} \mathbf{P}^{i, i}\left[Z^{N, 1}\right.$ and $Z^{N, 2}$ are not coalesced by time $\left.t N\right]=\frac{6 \sigma^{2}}{6 \sigma^{2}+\kappa}=\frac{\gamma}{\kappa}$.

This yields (2.21) of Lemma 2.2 and hence the statement of Lemma 2.2 holds also in the situation of long range interactions. Thus the proof of Theorem 2 is complete.

\section{REFERENCES}

[1] Baillon, J.-B., Clément, Ph., Greven, A. and den Hollander, F. (1995). On the attracting orbit of a non-linear transformation arising from renormalization of hierarchically interacting diffusions. I. The compact case. Canad. J. Math. 47 3-27.

[2] Cox, J. T., Durrett, R. and Perkins, E. A. (2000). Rescaled voter models converge to super-Brownian motion. Ann. Probab. 28 185-234.

[3] Cox, J. T., Fleischmann, K. and Greven, A. (1996). Comparison of interacting diffusions and an application to their ergodic theory. Probab. Theory Related Fields 105 513-528.

[4] CoX, J. T. and GREvEn, A. (1994). Ergodic theorems for infinite systems of locally interacting diffusions. Ann. Probab. 22 833-853.

[5] Dawson, D. A. (1993). Measure-valued Markov processes. École d'Été de Probabilités de Saint-Flour XXI. Lecture Notes in Math. 1541 1-260. Springer, Berlin.

[6] Durrett, R. and Perkins, E. A. (1999). Rescaled contact processes converge to superBrownian motion in two or more dimensions. Probab. Theory Related Fields 114309 399.

[7] Liggett, T. M. and Spitzer, F. (1981). Ergodic theorems for coupled random walks and other systems with locally interacting components. Z. Wahrsch. Verw. Gebiete $56443-$ 468.

[8] Mueller, C. and Tribe, R. (1995). Stochastic P.D.E.'s arising from the long range contact and long range voter processes. Probab. Theory Related Fields 102 519-545.

[9] Shiga, T. (1980). An interacting system in population genetics. J. Math. Kyoto Univ. 20213 242.

[10] Shiga, T. and ShimizU, A. (1980). Infinite-dimensional stochastic differential equations and their applications. J. Math. Kyoto Univ. 20 395-416. 